\title{
Application of a Revised Molière Theory to the Description of the Landau-Pomeranchuk Effect
}

\author{
Hrach Torosyan ${ }^{1}$, Olga Voskresenskaya ${ }^{2, *}$ \\ ${ }^{1}$ Laboratory of Nuclear Problems, Joint Institute for Nuclear Research, \\ Joliot-Curie 6, 141980, Dubna, Moscow region, Russia \\ ${ }^{2}$ Laboratory of Information Technologies, Joint Institute for Nuclear Research, \\ Joliot-Curie 6, 141980, Dubna, Moscow region, Russia \\ *Corresponding Author: voskr@jinr.ru
}

Copyright (c)2014 Horizon Research Publishing All rights reserved.

\begin{abstract}
Using a corrected value of the screening angular parameter in a revised Molière multiple scattering theory we have obtained analytically and numerically the Coulomb corrections to the quantities of the Migdal theory of the Landau-Pomeranchuk (LPM) effect for sufficiently thick targets. We showed that the Coulomb corrections to the spectral bremsstrahlung rate of this theory allow completely to eliminate the discrepancy between the theory and experiment for high $\mathrm{Z}$ experimental targets.
\end{abstract}

Keywords Landau-Pomeranchuk-Migdal effect, multiple scattering, Coulomb corrections

\section{Introduction}

The theory of the multiple scattering of charged particles has been treated by several authors [1-7]. However, the most widely used at present is the multiple scattering theory of Molière [3] whose results are employed nowadays in most of the transport codes. It is of interest for numerous applications related to particle transport in matter; and it also presents the most used tool for taking into account the multiple scattering effects in experimental data processing. The DIRAC experiment [8] like many others [9] (the MuScat [10], MUCOOL [11] experiments, etc.) meets the problem of the excluding of multiple scattering effects in matter from obtained data. The standard theory of multiple scattering [8, 9, 10], proposed by Molière [3] and Fano [6], and some its modifications [10, 12, 13] are used for this aim.

As the Molière theory is currently used roughly for $10-300 \mathrm{GeV}$ electron beams, the role of the high-energy corrections to the parameters of this theory becomes significant. Of especial importance is the Coulomb correction to the screening angular parameter, as this parameter also enters into other important quantities of the Molière theory.

Landau and Pomeranchuk were the first to show
[14] that multiplicity of electron scattering processes on atomic nuclei in an amorphous medium results in the suppression of soft bremsstrahlung. The quantitative theory of this phenomenon was created by Migdal $[15,16]^{1}$. Therefore, it received the name LandauPomeranchuk-Migdal (LPM) effect.

The analogous effects are possible also at coherent radiation of relativistic electrons and positrons in a crystalline medium [18], in cosmic-ray physics [19] (e.g. in applications motivated by extremely high energy IceCubes neutrino-induced showers with energies above 1 $\mathrm{PeV}[20])$. Effects of this kind should manifest themselves in scattering of protons on the nuclei, what has recently been shown in Groning by the AGOR collaboration [21], at penetration of quarks and partons through the nuclear matter at the RHIC and LHC energies [22]. The QCD analogue of the LPM effect was examined in [23]; a possibility studying the LPM effect in oriented crystal at GeV energy was analyzed in [24]. Theoretically, an analogue of the LPM effect was considered for nucleon-nucleon collisions in the neutron stars and supernovae [25], and also in relativistic plasmas [26].

The results of a series of experiments at the SLAC [27, 28, 29] and CERN-SPS [30, 31] accelerators on detection of the Landau-Pomeranchuk effect confirmed the basic qualitative conclusion that multiple scattering of ultrarelativistic charged particles in matter leads to suppression of their bremsstrahlung in the soft part of the spectrum. However, attempts to quantitatively describe the experimental data [27] faced an unexpected difficulty. For achieving satisfactory agreement of data with theory [15] the authors [27] had to multiply the results of their calculations in the Born approximation by a normalization factor $R$ equal to $0.94 \pm 0.01 \pm 0.032$, which had no reasonable explanation.

The alternate calculations $[32,33]$ gave a similar result despite different computational basis [27]. The theoretical predictions are agreement with the spectrum

\footnotetext{
${ }^{1}$ See also [17] accounting the edge effects. Let us notice that Molière's theory was not applied to the description of the LPM effect in these works. The previous results of the multiple scattering theory [2] were used here.
} 
of photon bremsstrahlung measured for $25 \mathrm{GeV}$ electron beam and $0.7-6.0 \% L_{R}^{2}$ gold targets over the range $30<\omega<500 \mathrm{MeV}$ of the emitted photon frequency $\omega$ only within a normalization factor 0.94 [27] - 0.93 [32]. The origin of the above small but significant disagreement between data and theory needs to be better understand [28].

In $[33,34,35]$ the multiphoton effects was taken into account, and a comparison with SLAC E-146 data was carried out. Nevertheless, the problem of normalization remained and is still not clear. The other authors, except $[32,35]$, do not discuss this normalization problem [29].

The aim of this work is to show that the discussed discrepancy can be explained at least for high $\mathrm{Z}$ targets if the corrections to the results of the Born approximation (i.e. the Coulomb corrections) are appropriately considered on the basis of a revised version of the Molière multiple scattering theory $[37,38]$.

The paper is organized as follows. In Section 2 we consider the basic formulae of the conventional [3] and a revised [37, 38] small-angle Molière multiple scattering theory applying in the next Section to the theory of the LPM effect and its analogue for a thin layer of an amorphous medium $[39,40]$. We also determine analytically and numerically the Coulomb corrections to some important parameters of the Molière theory for the target materials used in [28]. Then, in Section 3 we present the results of the Migdal LPM effect theory for sufficiently tick targets and obtain the analytical and numerical results for the Coulomb corrections to the quantities of this theory in regimes of the large and the small LPM suppression based on the Coulomb corrections found in Section 2. Additionally, we obtain numerical results for Coulomb corrections to the asymptotes of the spectral radiation rate within the LPM theory analogue for a thin target. Finally, in Section 4 we briefly sum up our results.

\section{Molière's multiple scattering theory}

\subsection{Conventional Molière's theory}

Let $w_{M}(\vartheta, L)$ be a spatial-angle particle distribution function in a homogenous medium, and $\boldsymbol{\vartheta}$ is a twodimensional particle scattering angle in the plane orthogonal to the incident particle direction. For smallangle approximation $\vartheta \ll 1(\sin \vartheta \sim \vartheta)$, the above distribution function is the number of particles scattered in the angular interval $d \vartheta$ after traveling through the target of thickness $L$. In the notation of Molière, it reads

$$
w_{M}(\vartheta, L)=\int_{0}^{\infty} J_{0}(\vartheta \eta) \exp \left[-n_{0} L \cdot \nu(\eta)\right] \eta d \eta
$$

where

$$
\nu(\eta)=2 \pi \int_{0}^{\infty} \sigma_{0}(\boldsymbol{\theta})\left[1-J_{0}(\theta \eta)\right] \boldsymbol{\theta} d \boldsymbol{\theta}
$$

\footnotetext{
${ }^{2} L_{\mathrm{R}}$ is the radiation length of the target material.
}

$J_{0}(\vartheta \eta)$ is the Bessel function, $n_{0}$ denotes the number density, $\sigma_{0}(\boldsymbol{\theta})$ presents the Born differential scattering cross-section, and $\boldsymbol{\theta}=\boldsymbol{\vartheta}^{\prime}-\boldsymbol{\vartheta}$.

The function (1) satisfies the well-known Boltzmann transport equation, written here with the small angle approximation

$$
\begin{gathered}
\frac{\partial w(\vartheta, L)}{\partial L}=-n_{0} w_{M}(\vartheta, L) \int \sigma_{0}(\boldsymbol{\theta}) d^{2} \boldsymbol{\theta} \\
+n_{0} \int w_{M}(\boldsymbol{\vartheta}+\boldsymbol{\theta}, L) \sigma_{0}(\boldsymbol{\theta}) d^{2} \boldsymbol{\theta} \\
=n_{0} \int\left[w_{M}(\boldsymbol{\vartheta}+\boldsymbol{\theta}, L)-w_{M}(\vartheta, L)\right] \sigma_{0}(\boldsymbol{\theta}) d^{2} \boldsymbol{\theta} .
\end{gathered}
$$

The Gaussian particle distribution function used in the Migdal LPM effect theory, which differs from (1), can be derived from the Boltzmann transport equation by the Fokker-Plank method [41].

One of the most important results of the Molière theory is that the scattering is described by a single parameter, the so-called screening angle $\left(\theta_{a}\right.$ or $\left.\theta_{a}^{\prime}\right)$

$$
\theta_{a}^{\prime}=\sqrt{1.167} \theta_{a}=\left[\exp \left(C_{E}-0.5\right)\right] \theta_{a} \approx 1.080 \theta_{a},
$$

where $C_{E}=0.577 \ldots$ is the Euler constant.

More precisely, the angular distribution depends only on the logarithmic ratio $b$

$$
b=\ln \left(\frac{\theta_{c}}{\theta_{a}^{\prime}}\right)^{2} \equiv \ln \left(\frac{\theta_{c}}{\theta_{a}}\right)^{2}+1-2 C_{E}
$$

of the characteristic angle $\theta_{c}$ describing the foil thickness

$$
\theta_{c}^{2}=4 \pi n_{0} L\left(\frac{Z \alpha}{\beta p}\right)^{2}, \quad p=m v
$$

to the screening angle $\theta_{a}^{\prime}$, which characterizes the scattering atom.

In order to obtain a result valid for large angles, Molière defines a new parameter $B$ by the transcendental equation

$$
B-\ln B=b
$$

The angular distribution function can be written then as

$$
\begin{aligned}
& w_{M}(\vartheta, B)=\frac{1}{\overline{\vartheta^{2}}} \int_{0}^{\infty} y d y J_{0}(\vartheta y) e^{-y^{2} / 4} \\
& \times \exp \left[\frac{y^{2}}{4 B} \ln \left(\frac{y^{2}}{4}\right)\right], \quad y=\theta_{c} \eta
\end{aligned}
$$

The Molière expansion method is to consider the term $y^{2} \ln \left(y^{2} / 4\right) / 4 B$ as a small parameter. Then, the angular distribution function is expanded in a power series in $1 / B$

$$
w_{M}(\vartheta, L)=\sum_{n=0}^{\infty} \frac{1}{n !} \frac{1}{B^{n}} w_{n}(\vartheta, L)
$$

in which

$$
w_{n}(\vartheta, L)=\frac{1}{\overline{\vartheta^{2}}} \int_{0}^{\infty} y d y J_{0}\left(\frac{\vartheta}{\bar{\vartheta}} y\right) e^{-y^{2} / 4}
$$




$$
\begin{gathered}
\times\left[\frac{y^{2}}{4} \ln \left(\frac{y^{2}}{4}\right)\right]^{n}, \\
\overline{\vartheta^{2}}=\theta_{c}^{2} B=4 \pi n_{0} L\left(\frac{Z \alpha}{\beta p}\right)^{2} B(L) .
\end{gathered}
$$

This method is valid for $B>4.5$ and $\overline{\vartheta^{2}}<1$.

The first function $w_{0}(\vartheta, L)$ has a simple analytical form

$$
\begin{gathered}
w_{0}(\vartheta, L)=\frac{2}{\overline{\vartheta^{2}}} \exp \left(-\frac{\vartheta^{2}}{\overline{\vartheta^{2}}}\right), \\
\overline{\vartheta^{2}} \underset{L \rightarrow \infty}{\sim} \frac{L}{L_{R}} \ln \left(\frac{L}{L_{R}}\right),
\end{gathered}
$$

where For small angles, i.e. $\vartheta / \bar{\vartheta}=\vartheta /\left(\theta_{c} \sqrt{B}\right)$ less than about 2, the Gaussian (12) is the dominant term. In this region, $w_{1}(\vartheta, L)$ is in general less than $w_{0}(\vartheta, L)$, so that the corrections to the Gaussian is of order of $1 / B$, i.e. about $10 \%$.

A good approximate representation of the distribution at any angle is

$$
w_{M}(\vartheta, L) \approx w_{0}(\vartheta, L)+\frac{1}{B} w_{1}(\vartheta, L)
$$

with

$$
\begin{aligned}
w_{1}(\vartheta, L)= & \frac{1}{\overline{\vartheta^{2}}} \int_{0}^{\infty} y d y J_{0}\left(\frac{\vartheta}{\sqrt{\vartheta^{2}}} y\right) e^{-y^{2} / 4} \\
& \times\left[\frac{y^{2}}{4} \ln \left(\frac{y^{2}}{4}\right)\right] .
\end{aligned}
$$

This approximation was applied by authors of [40] to the analysis of data $[27,28]$ over the region $\omega<30 \mathrm{MeV}$ that will be shown in Section 3 .

As show the classical works of Molière [3], the quantity (2) can be represented in the area of the important $\eta$ values $0 \leq \eta \leq 1 / \theta_{c}$ as

$$
\nu(\eta)=-4 \pi\left(\frac{Z \alpha}{\beta p}\right)^{2} \eta^{2}\left[\ln \left(\frac{\eta \theta_{a}}{2}\right)+C_{E}-\frac{1}{2}\right]
$$

where the screening angle $\theta_{a}$ depends both on the screening properties of the atom and on the $\sigma_{0}(\boldsymbol{\theta})$ approximation used for its calculation.

Using the Thomas-Fermi model of the atom and an interpolation scheme, Molière obtained $\theta_{a}$ for the cases where $\sigma_{0}(\boldsymbol{\theta})$ is calculated within the Born and quasiclassical approximations:

$$
\begin{gathered}
\theta_{a}^{B}=1.20 \cdot \alpha \cdot Z^{1 / 3} \\
\theta_{a}^{M}=\theta_{a}^{B} \sqrt{1+3.34 \cdot(Z \alpha / \beta)^{2}} .
\end{gathered}
$$

Here, $Z$ is the nuclear charge number of the target atom, $\alpha=1 / 137$ is the fine structure constant, and $\beta=v / c$ is the velocity of a projectile in units of the velocity of light.

The latter result (18) is only approximate (see critical remarks on its derivation in [41]). Below we will present an exact analytical and numerical result for the screening angular parameter.

\subsection{Revised multiple scattering theory of Molière}

Very recently, it was shown [38] that for any model of the atom the following rigorous relation determining the screening angular parameter $\theta_{a}^{\prime}$ is valid:

$$
\ln \left(\theta_{a}^{\prime}\right)=\ln \left(\theta_{a}^{\prime}\right)^{B}+\operatorname{Re}[\psi(1+i Z \alpha / \beta)]+C_{E}
$$

or, equivalently,

$$
\Delta_{C C}\left[\ln \left(\theta_{a}^{\prime}\right)\right] \equiv \ln \left(\theta_{a}^{\prime}\right)-\ln \left(\theta_{a}^{\prime}\right)^{B}=f(Z \alpha / \beta),
$$

where $\Delta_{C C}$ is the Coulomb correction to the Born result, $\psi$ is the logarithmic derivative of the gamma function $\Gamma$, and $f(Z \alpha / \beta)$ is an universal function of the Born parameter $\xi=Z \alpha / \beta$, which is also known as the BetheMaximon function:

$$
f(\xi)=\xi^{2} \sum_{n=1}^{\infty} \frac{1}{n\left(n^{2}+\xi^{2}\right)} .
$$

To compare the approximate Molière result (18) with the exact one (20), we first present (18) in the form

$$
\delta_{M}\left[\theta_{a}\right] \equiv \frac{\theta_{a}^{M}-\theta_{a}^{B}}{\theta_{a}^{B}}=\sqrt{1+3.34 \xi^{2}}-1
$$

and also rewrite (20) as follows:

$$
\begin{aligned}
\delta_{C C}\left[\theta_{a}\right] & \equiv \frac{\theta_{a}-\theta_{a}^{B}}{\theta_{a}^{B}}=\frac{\theta_{a}^{\prime}-\left(\theta_{a}^{\prime}\right)^{B}}{\left(\theta_{a}^{\prime}\right)^{B}} \\
& =\exp [f(\xi)]-1 .
\end{aligned}
$$

Then we get:

$$
\delta_{C C M}[\delta] \equiv-\frac{\delta_{C C}\left[\theta_{a}\right]-\delta_{M}\left[\theta_{a}\right]}{\delta_{M}\left[\theta_{a}\right]}=\frac{\Delta_{C C M}[\delta]}{\delta_{M}\left[\theta_{a}\right]} .
$$

In order to obtain relative difference between the approximate $\theta_{a}^{\mathrm{M}}$ and exact $\theta_{a}$ results for the screening angle

$$
\begin{aligned}
\delta_{\mathrm{CCM}}\left[\theta_{a}\right] & \equiv \frac{\theta_{a}-\theta_{a}^{\mathrm{M}}}{\theta_{a}^{\mathrm{M}}}=\frac{\theta_{a}}{\theta_{a}^{\mathrm{M}}}-1 \\
& =R_{\mathrm{CCM}}\left[\theta_{a}\right]-1,
\end{aligned}
$$

we rewrite (22) and (23) in the following form

$$
\delta_{\mathrm{CC}}\left[\theta_{a}\right]+1=\frac{\theta_{a}}{\theta_{a}^{\mathrm{B}}}, \quad \delta_{\mathrm{M}}\left[\theta_{a}\right]+1=\frac{\theta_{a}^{\mathrm{M}}}{\theta_{a}^{\mathrm{B}}}
$$

and obtain for the ratio $R_{\mathrm{CCM}}\left[\theta_{a}\right]$ the expression

$$
\begin{aligned}
R_{\mathrm{CCM}}\left[\theta_{a}\right] & \equiv \frac{\theta_{a}}{\theta_{a}^{\mathrm{M}}}=\frac{\delta_{\mathrm{CC}}\left[\theta_{a}\right]+1}{\delta_{\mathrm{M}}\left[\theta_{a}\right]+1} \\
= & \delta_{\mathrm{CCM}}\left[\theta_{a}\right]+1 .
\end{aligned}
$$

We can also represent the relative difference (25) by the equation

$$
\delta_{\mathrm{CCM}}\left[\theta_{a}\right]=\frac{\Delta_{\mathrm{CCM}}[\delta]}{\delta_{\mathrm{M}}\left[\theta_{a}\right]+1} .
$$


Table 1. Numerical results for the relative corrections (22), (23), relative differences (24), (26), and the ratio (28) in the range of nuclear charge $73 \leq Z \leq 92$.

\begin{tabular}{cccccc}
\hline $\mathrm{Z}$ & $\delta_{\mathrm{M}}\left[\theta_{a}\right]$ & $\delta_{\mathrm{CC}}\left[\theta_{a}\right]$ & $\delta_{\mathrm{CCM}}[\delta]$ & $\delta_{\mathrm{CCM}}\left[\theta_{a}\right]$ & $R_{\mathrm{CCM}}\left[\theta_{a}\right]$ \\
\hline 73 & 0.396 & 0.318 & -0.198 & -0.056 & 0.944 \\
74 & 0.404 & 0.325 & -0.196 & -0.056 & 0.943 \\
78 & 0.443 & 0.359 & -0.189 & -0.058 & 0.942 \\
79 & 0.452 & 0.367 & -0.188 & -0.059 & 0.941 \\
82 & 0.482 & 0.393 & -0.185 & -0.060 & 0.940 \\
92 & 0.583 & 0.485 & -0.169 & -0.062 & 0.938 \\
\hline
\end{tabular}

For some high $\mathrm{Z}$ targets used in [28] and $\beta=1$, we obtain the following values of the relative Molière $\delta_{\mathrm{M}}\left[\theta_{a}\right]$ (22) and Coulomb $\delta_{\mathrm{CC}}\left[\theta_{a}\right](23)$ corrections and also the sizes of the difference $\Delta_{\mathrm{CCM}}[\delta]$ and relative differences $\delta_{\mathrm{CCM}}[\delta](24), \delta_{\mathrm{CCM}}\left[\theta_{a}\right](26)$ as well as the ratio $R_{\mathrm{CCM}}\left[\theta_{a}\right]$ (28) (Table 1, Figure 1).

From the Table 1 it is evident that the Coulomb correction $\delta_{\mathrm{CC}}\left[\theta_{a}\right]$ has a large value, which ranges from around $30 \%$ for $Z \sim 70$ up to $50 \%$ for $Z \sim 90$. The relative difference between the approximate and exact results for this Coulomb correction varies from 17 up to $20 \%$ over the range $73 \leq Z \leq 92$.

The relative difference $\delta_{C C M}\left[\theta_{a}\right]$ between the approximate $\theta_{a}^{\mathrm{M}}$ and exact $\theta_{a}$ results for the screening angle as well as $R_{C C M}\left[\theta_{a}\right]=\theta_{a} / \theta_{a}^{\mathrm{M}}$ value does not vary significantly from one target material to another. Their sizes are $5.86 \pm 0.22 \%$ for $-\delta_{\mathrm{CCM}}\left[\theta_{a}\right]$ and $0.941 \pm 0.002$ for $R_{\mathrm{CCM}}\left[\theta_{a}\right]$ in the $\mathrm{Z}$ range studied. It is interesting that the latter value coincides with the normalization constant $R=0.94 \pm 0.01$ found in [27].

We show further that the aforesaid discrepancy between theory of the LPM effect and experiment [27, 28, 32 ] can be completely eliminated for heavy target elements on the basis of the Coulomb corrections to the screening angular parameter. For this purpose, we calculate now some additional Coulomb corrections to other important parameters of the Molière theory. Inserting (5) into (7) and differentiating the latter, we arrive at

$$
\Delta_{C C}[b]=-f(\xi)=\left(1-\frac{1}{B^{B}}\right) \cdot \Delta_{C C}[B] .
$$

So $\Delta_{C C}[B]$ becomes

$$
\Delta_{C C}[B]=\frac{f(\xi)}{1 / B^{B}-1} .
$$

Accounting $\overline{\vartheta^{2}}=\theta_{c}^{2} B(11)$, we get

$$
\Delta_{C C}\left[\overline{\vartheta^{2}}\right] \equiv \overline{\vartheta^{2}}-\left(\overline{\vartheta^{2}}\right)^{B}=\theta_{c}^{2} \cdot \Delta_{C C}[B] \text {. }
$$

Finally, the relative Coulomb corrections can be represented as

$$
\delta_{C C}\left[\overline{\vartheta^{2}}\right]=\delta_{C C}[B]=\frac{f(\xi)}{1-B^{B}}
$$

The Z dependence of the corrections (31), (32), and (34) presents Table 2 (see also Figure 1).
Table 2. The Coulomb correction (31), (32), and (34) to the parameters of the Molière theory for $B^{B}=8.46$ and $\beta=1$.

\begin{tabular}{cccccc}
\hline $\mathrm{M}$ & $\mathrm{Z}$ & $\Delta_{C C}[b]$ & $\Delta_{C C}[B]$ & $\delta_{C C}[B]$ & $\delta_{C C}\left[\overline{\vartheta^{2}}\right]$ \\
\hline $\mathrm{Al}$ & 13 & -0.0107 & -0.0121 & -0.0014 & -0.0014 \\
$\mathrm{Fe}$ & 26 & -0.0420 & -0.0476 & -0.0056 & -0.0056 \\
$\mathrm{~W}$ & 74 & -0.2813 & -0.3190 & -0.0377 & -0.0377 \\
$\mathrm{Au}$ & 79 & -0.3125 & -0.3545 & -0.0419 & -0.0419 \\
$\mathrm{~Pb}$ & 82 & -0.3316 & -0.3760 & -0.0445 & -0.0445 \\
$\mathrm{U}$ & 92 & -0.3951 & -0.4481 & -0.0530 & -0.0530 \\
\hline
\end{tabular}

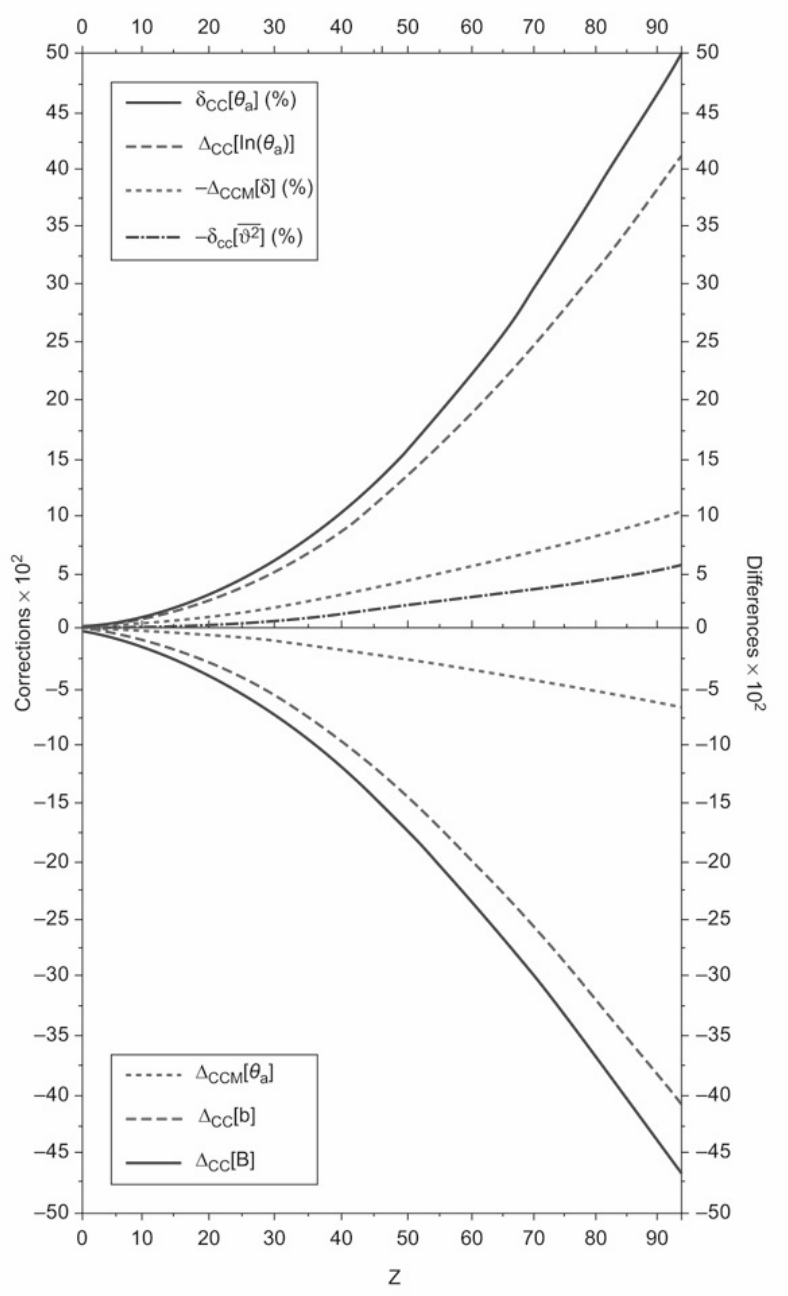

Figure 1. The $Z$ dependence of the Coulomb corrections to some parameters of the Moliere theory and the differences between exact and approximate results [38].

\section{Applications of the Molière theory to the description of the LPM effect and its analogue}

\subsection{Basic formulae of the Migdal LPM effect theory for sufficiently thick targets}

There exist two methods that allow to develop a rigorous quantitative theory of the Landau-Pomeranchuk 
effect. It is Migdal's method of kinetic equation [15, 16] and the method of functional integration $[32,33,35,36$, 42]. Neglecting numerically small quantum-mechanical corrections, we will adhere to version of the LandauPomeranchuk effect theory developed in [15].

Simple though quite cumbersome calculations yield the following formula for the electron spectral bremsstrahlung intensity averaged over various trajectories of electron motion in an amorphous medium (hereafter the units $\hbar=c=1, e^{2}=1 / 137$ are used):

$$
\left\langle\frac{d I}{d \omega}\right\rangle=\Phi(s)\left(\frac{d I}{d \omega}\right)_{0}
$$

where $(d I / d \omega)_{0}$ is the spectral bremsstrahlung rate without accounting the multiple scattering effects in the radiation,

$$
\begin{gathered}
\left(\frac{d I}{d \omega}\right)_{0}=\frac{2 e^{2}}{3 \pi} \gamma^{2} q L, \\
q=\overline{\vartheta^{2}} / L,
\end{gathered}
$$

and $\gamma$ is the Lorentz factor of the scattered particle.

The function $\Phi(s)$ accounts the multiple scattering influence on the bremsstrahlung rate and reads

$$
\begin{gathered}
\Phi(s)=24 s^{2}\left[\int_{0}^{\infty} d x e^{-2 s x} \operatorname{cth}(x) \sin (2 s x)-\frac{\pi}{4}\right], \\
s^{2}=\lambda^{2} / \overline{\vartheta^{2}}, \quad \lambda^{2}=\gamma^{-2} .
\end{gathered}
$$

It has simple asymptotes at the small and large values of the argument:

$$
\begin{gathered}
\Phi(s) \rightarrow\left\{\begin{array}{cc}
6 s, & s \rightarrow 0 \\
1, & s \rightarrow \infty
\end{array}\right. \\
s=\frac{1}{4 \gamma^{2}} \sqrt{\frac{\omega}{q}} .
\end{gathered}
$$

For $s \ll 1$, the suppression is large, and $\Phi(s) \approx 6 s$. The intensity of radiation in this case is much less, than the corresponding result of Bethe and Heitler. If $s \geq 1$, the function $\Phi(s)$ is close to a unit, and the following approximation is valid [18]:

$$
\Phi(s) \approx 1-0.012 / s^{4} .
$$

The formula (35) is obtained with the logarithmic accuracy. At $s \gg 1,(35)$ coincides within this accuracy with the Bethe-Heitler result

$$
\left\langle\frac{d I}{d \omega}\right\rangle_{B H}=\frac{L}{L_{R}}\left[1+\frac{1}{12 \ln \left(183 Z^{-1 / 3}\right)}\right] .
$$

If $s \ll 1$, we have the large LPM suppression in comparison with (43). Let us notice that effect of a medium polarization is not considered here, i.e. it is assumed that the absolute permittivity of the medium $\varepsilon(\omega)=1$.

\subsection{Applying the revised theory of Molière to the Migdal LPM effect theory}

Now we obtain analytical and numerical results for the Coulomb corrections to the quantities of the Migdal LPM effect theory. In order to derive an analytical expression for the Coulomb correction to the Born spectral bremsstrahlung rate $(d I / d \omega)_{0}$, we first write

$$
\begin{gathered}
\Delta_{C C}\left[\left(\frac{d I}{d \omega}\right)_{0}\right] \equiv\left(\frac{d I}{d \omega}\right)_{0}-\left(\frac{d I}{d \omega}\right)_{0}^{B} \\
=\frac{2 e^{2}}{3 \pi} \gamma^{2} L \cdot \Delta_{C C}[q]
\end{gathered}
$$

where

$$
\begin{gathered}
\Delta_{C C}[q] \equiv q-q^{B}=\frac{1}{L} \cdot \Delta_{C C}\left[\overline{\vartheta^{2}}\right], \\
\Delta_{C C}\left[\overline{\vartheta^{2}}\right] \equiv \overline{\vartheta^{2}}-\left(\overline{\vartheta^{2}}\right)^{B}=\theta_{c}^{2} \cdot \Delta_{C C}[B], \\
\Delta_{C C}[B]=\frac{f(\xi)}{1 / B^{B}-1} .
\end{gathered}
$$

In doing so, (44) becomes

$$
\Delta_{C C}\left[\left(\frac{d I}{d \omega}\right)_{0}\right]=\frac{2\left(e \gamma \theta_{c}\right)^{2}}{3 \pi\left(1 / B^{B}-1\right)} \cdot f(\xi),
$$

and the relative Coulomb correction reads

$$
\begin{gathered}
\delta_{C C}\left[(d I / d \omega)_{0}\right]=\delta_{C C}[q]=\delta_{C C}\left[\overline{\vartheta^{2}}\right] \\
=R_{C C}\left[(d I / d \omega)_{0}\right]-1=\frac{f(\xi)}{1-B^{B}} .
\end{gathered}
$$

Next, in order to obtain the relative Coulomb correction to the Migdal function $\Phi(s)$, we first derive corresponding correction to the parameter $s^{2}(39)$ :

$$
\begin{gathered}
\Delta_{C C}\left[s^{2}\right]=\frac{\omega}{16 \gamma^{4}}\left(\frac{1}{q}-\frac{1}{q^{B}}\right), \\
\delta_{C C}\left[s^{2}\right]=\frac{q^{B}}{q}-1=\frac{\left(\overline{\vartheta^{2}}\right)^{B}}{\overline{\vartheta^{2}}}-1, \\
\frac{\left(\overline{\vartheta^{2}}\right)^{B}}{\overline{\vartheta^{2}}}=\frac{1}{\delta_{C C}\left[\overline{\vartheta^{2}}\right]+1} .
\end{gathered}
$$

This leads to the following relative Coulomb correction for $s(41)$ :

$$
\begin{aligned}
& \delta_{C C}[s]=\sqrt{\frac{\left(\overline{\vartheta^{2}}\right)^{B}}{\overline{\vartheta^{2}}}}-1 \\
& =\frac{1}{\sqrt{\delta_{C C}\left[\overline{\vartheta^{2}}\right]+1}}-1 .
\end{aligned}
$$

For the asymptote $\Phi(s)=6 s(40)$, we get

$$
\delta_{C C}[\Phi(s)]=\delta_{C C}[s]=\frac{1}{\sqrt{R_{C C}\left[(d I / d \omega)_{0}\right]}}-1 .
$$

Then the total relative Coulomb correction to the spectral density of radiation in this asymptotic case becomes

$$
\delta_{C C}[\langle d I / d \omega\rangle]=\delta_{C C}\left[(d I / d \omega)_{0}\right]+\delta_{C C}[\Phi(s)] .
$$

Table 3 gives the numerical values of these Coulomb corrections for $B^{B}=8.45$ [40] corresponding to the conditions of experiment [27]. 
Table 3.

Coulomb corrections to the quantities of the Migdal LPM theory $\delta_{C C}\left[(d I / d \omega)_{0}\right](47), \delta_{C C}[\Phi(s)](52)$, and $\delta_{C C}[\langle d I / d \omega\rangle](53)$ in the regime of strong LPM suppression for high $\mathrm{Z}$ target materials of the experiment $[27]^{3}, B^{B}=8.46$, and $\beta=1$.

\begin{tabular}{ccccc}
\hline $\mathrm{Z}$ & $\delta_{C C}\left[\left(\frac{d I}{d \omega}\right)_{0}\right]$ & $\delta_{C C}[\Phi(s)]$ & $\delta_{C C}\left[\left\langle\frac{d I}{d \omega}\right\rangle\right]$ & $R_{C C}\left[\left\langle\frac{d I}{d \omega}\right\rangle\right]$ \\
\hline 74 & -0.0377 & -0.0194 & -0.0571 & 0.943 \\
79 & -0.0419 & -0.0216 & -0.0635 & 0.936 \\
82 & -0.0445 & -0.0230 & -0.0675 & 0.932 \\
92 & -0.0530 & -0.0276 & -0.0806 & 0.919 \\
\hline
\end{tabular}

It shows that the ratio $R_{C C}[\langle d I / d \omega\rangle](74 \leq Z \leq$ 92 ) is formally close to the normalization factor $R=$ $0.94 \pm 0.01 \pm 0.032$ from [27] for heavy target elements. However, the regime of strong LPM suppression is not reached in the conditions of the experiment [27, 28, 29]. Therefore, we will carry out now calculation for the regime of small LPM suppression (42).

In order to obtain the relative correction $\delta_{\mathrm{CC}}[\Phi(s)]$ in this regime, we first derive an expression for the Coulomb correction $\Delta_{\mathrm{CC}}[\Phi(s)]$ to the Migdal function $\Phi(s)$ :

$$
\begin{gathered}
\Delta_{\mathrm{CC}}[\Phi(s)]=0.012\left(\frac{1}{\left(s^{4}\right)^{\mathrm{B}}}-\frac{1}{s^{4}}\right)=\frac{0.012}{s^{4}} \delta_{\mathrm{CC}}\left[s^{4}\right], \\
\delta_{\mathrm{CC}}\left[s^{4}\right]=\left(\frac{q^{\mathrm{B}}}{q}\right)^{2}-1=\left(\frac{\left(\overline{\vartheta^{2}}\right)^{\mathrm{B}}}{\overline{\vartheta^{2}}}\right)^{2}-1 \\
=\frac{1}{\left(\delta_{\mathrm{CC}}\left[\overline{\vartheta^{2}}\right]+1\right)^{2}}-1=\frac{1}{\left(R_{\mathrm{CC}}\left[(d I / d \omega)_{0}\right]\right)^{2}}-1 .
\end{gathered}
$$

This leads to the following relative Coulomb correction for $\Phi(s)(42)$ :

$$
\begin{gathered}
\delta_{\mathrm{CC}}[\Phi(s)]=\frac{0.012}{s^{4}} \delta_{\mathrm{CC}}\left[s^{4}\right] \cdot \frac{\left(s^{4}\right)^{\mathrm{B}}}{\left(s^{4}\right)^{\mathrm{B}}-0.012} . \\
=0.012 \frac{\delta_{\mathrm{CC}}\left[s^{4}\right]}{\delta_{\mathrm{CC}}\left[s^{4}\right]+1} \cdot \frac{1}{\left(s^{4}\right)^{\mathrm{B}}-0.012} .
\end{gathered}
$$

In Table 4 are listed the values of the relative Coulomb corrections to the quantities of (35) in the regime of small LPM suppression (42) for some separate $s$ values from the range $1.0 \leq s \leq \infty$ (for instance, $s=1.1$ and $s=1.5)$.

Table 5 presents the average values of the corrections $-\delta_{C C}[\langle d I / d \omega\rangle](\%)$ for separate high $\mathrm{Z}$ target elements and the common average $-\bar{\delta}_{C C}[\langle d I / d \omega\rangle](\%)$ over the entire range $1.0 \leq s \leq \infty$ of the parameter $s$, for which the regime of small LPM suppression is valid.

It will be seen from Table 5 that the Coulomb corrections $\delta_{C C}[\langle d I / d \omega\rangle]=-4.50 \pm 0.05 \%(Z=82)$ and $\delta_{C C}[\langle d I / d \omega\rangle]=-5.35 \pm 0.06 \%(Z=92)$ coincide within the experimental error with the sizes of the normalization correction $-4.5 \pm 0.2 \%$ for $2 \% L_{R}$ lead target and

\footnotetext{
${ }^{3}$ For low $\mathrm{Z}$ targets, the E-146 data showed a disagreement with the Migdal LPM theory predictions. There is a problem of an adequate describe the photon spectra shape for the low $\mathrm{Z}$ targets $[28,29]$. Therefore, we will analyze only results for some high $\mathrm{Z}$ targets of the SLAC E-146 experiment.
}

$-5.6 \pm 0.3 \%$ for $3 \% L_{R}$ uranium target, respectively (Table II in [28]).

Table 4. Coulomb corrections to the quantities of the Migdal LPM theory, $\delta_{C C}\left[(d I / d \omega)_{0}\right](47), \delta_{C C}[\Phi(s)]$ $(55)$, and $\delta_{C C}[\langle d I / d \omega\rangle](53)$, in the regime of small LPM suppression for high $\mathrm{Z}$ targets of experiment [28] 1. at $\beta=1, B^{B}=8.46$, and $s=1.1$

\begin{tabular}{ccccc}
\hline $\mathrm{Z}$ & $\delta_{C C}\left[\left(\frac{d I}{d \omega}\right)_{0}\right]$ & $\delta_{\mathrm{CC}}\left[s^{4}\right]$ & $\delta_{C C}[\Phi(s)]$ & $\delta_{C C}\left[\left\langle\frac{d I}{d \omega}\right\rangle\right]$ \\
\hline 79 & -0.0419 & -0.0896 & -0.0008 & -0.0427 \\
82 & -0.0445 & -0.0953 & -0.0009 & -0.0454 \\
92 & -0.0530 & -0.1149 & -0.0011 & -0.0541 \\
\hline
\end{tabular}

$$
\bar{\delta}_{C C}[\langle d I / d \omega\rangle]=-4.74 \pm 0.59 \%
$$

2. at $\beta=1, B^{B}=8.46$, and $s=1.5$

\begin{tabular}{ccccc}
\hline $\mathrm{Z}$ & $\delta_{C C}\left[\left(\frac{d I}{d \omega}\right)_{0}\right]$ & $\delta_{\mathrm{CC}}\left[s^{4}\right]$ & $\delta_{C C}[\Phi(s)]$ & $\delta_{C C}\left[\left\langle\frac{d I}{d \omega}\right\rangle\right]$ \\
\hline 79 & -0.0419 & -0.0896 & -0.0002 & -0.0421 \\
82 & -0.0445 & -0.0953 & -0.0002 & -0.0447 \\
92 & -0.0530 & -0.1149 & -0.0003 & -0.0533 \\
\hline
\end{tabular}

$$
\bar{\delta}_{C C}[\langle d I / d \omega\rangle]=-4.67 \pm 0.57 \% .
$$

Table 5. The dependence of the relative Coulomb correction $-\delta_{C C}[\langle d I / d \omega\rangle]$ value $(\%)$ on the parameter $s$ in the regime of small LPM suppression for high $\mathrm{Z}$ targets, $\beta=1$, and $B^{B}=8.46$.

\begin{tabular}{cccccccc}
\hline$Z \backslash s$ & 1.0 & 1.1 & 1.2 & 1.3 & 1.5 & 2.0 & $\infty$ \\
\hline 79 & 4.32 & 4.28 & 4.26 & 4.24 & 4.22 & 4.21 & 4.19 \\
82 & 4.58 & 4.54 & 4.51 & 4.49 & 4.47 & 4.46 & 4.45 \\
92 & 5.45 & 5.41 & 5.36 & 5.34 & 5.33 & 5.31 & 5.30 \\
\hline
\end{tabular}

$$
\begin{gathered}
\delta_{C C}[\langle d I / d \omega\rangle]=-4.50 \pm 0.05 \%(Z=82) \\
\delta_{C C}[\langle d I / d \omega\rangle]=-5.35 \pm 0.06 \%(Z=92) \\
\bar{\delta}_{C C}[\langle d I / d \omega\rangle]=-4.70 \pm 0.49 \%
\end{gathered}
$$

It is also obvious that the average $\delta_{C C}[\langle d I / d \omega\rangle]$ value $\bar{\delta}_{C C}[\langle d I / d \omega\rangle]=-4.70 \pm 0.49 \%$ excellent agrees with the weighted average $-4.7 \pm 2 \%$ of the normalization correction obtained in [28] for $25 \mathrm{GeV}$ data $^{4}$.

We believe that this allows to understand an origin of the discussed in $[27,28]$ normalization problem for high $\mathrm{Z}$ targets.

\subsection{Application of Molière's theory to the de- scription of the LPM effect analogue for a thin target}

Experiment [27, 28] caused considerable interest and stimulated development of various approaches to the study of the LPM effect, including an application of Molière's results to the description of an analogue of the LPM effect for a thin layer of matter $[40]^{5}$.

\footnotetext{
${ }^{4}$ It becomes $-4.8 \pm 3.5 \%$ for the $8 \mathrm{GeV}$ data if the outlying $6 \% L_{R}$ gold target is excluded from them [28].

${ }^{5}$ The authors of [40] neglect the influence of the medium polarization [43] on the radiation in this theory.
} 
In [40] it is shown that the region of the emitted photon frequencies naturally splits into two intervals, $\omega>\omega_{c}$ and $\omega<\omega_{c}$, in first of which the LPM effect for sufficiently tick targets takes place, and in the second, there is its analogue for thin targets. The quantity $\omega_{c}$ is defined here as $\omega_{c}=2 \gamma^{2} / L$.

Application of the Molière multiple scattering theory to the analysis of experimental data $[27,28]$ for a thin target in the second $\omega$ range is based on the use of the expression for the spatial-angle particle distribution function (1), which satisfies the standard Boltzmann transport equation for a thin homogenous foil and differs significantly from the Gaussian particle distribution of the Migdal LPM effect theory.

Besides, it determines an another expression for the spectral radiation rate in the context of the coherent radiation theory [40], which reads

$$
\left\langle\frac{d I}{d \omega}\right\rangle=\int w_{M}(\vartheta) \frac{d I(\vartheta)}{d \omega} d^{2} \vartheta
$$

Here

$$
\frac{d I(\vartheta)}{d \omega}=\frac{2 e^{2}}{\pi}\left[\frac{2 \chi^{2}+1}{\chi \sqrt{\chi^{2}+1}} \ln \left(\chi+\sqrt{\chi^{2}+1}\right)-1\right]
$$

with $\chi=\gamma \vartheta / 2$. The latter expression is valid for consideration of the particle scattering in both amorphous and crystalline medium.

The formula (57) has simple asymptotes at the small and large values of parameter $\chi$ :

$$
\frac{d I(\vartheta)}{d \omega}=\frac{2 e^{2}}{3 \pi}\left\{\begin{array}{cc}
\gamma^{2} \vartheta^{2}, & \gamma \vartheta \ll 1, \\
3\left[\ln \left(\gamma^{2} \vartheta^{2}\right)-1\right], & \gamma \vartheta \gg 1,
\end{array}\right.
$$

Replacing in this formula $\vartheta^{2}$ by the average square value of the scattering angle $\overline{\vartheta^{2}}$, we arrive at the following estimates for the average radiation spectral density value:

$$
\left\langle\frac{d I}{d \omega}\right\rangle=\frac{2 e^{2}}{3 \pi}\left\{\begin{array}{cl}
\gamma^{2} \overline{\vartheta^{2}}, & \gamma^{2} \overline{\vartheta^{2}} \ll 1, \\
3\left[\ln \left(\gamma^{2} \overline{\vartheta^{2}}\right)-1\right], & \gamma^{2} \overline{\vartheta^{2}} \gg 1 .
\end{array}\right.
$$

In the experiment $[27,28]$, the above frequency intervals correspond roughly to the following $\omega$ ranges: $\left(\omega>\omega_{c}\right) \sim(\omega>30 \mathrm{MeV})$ and $\left(\omega<\omega_{c}\right) \sim(\omega<$ $30 \mathrm{MeV}$ ) for $25 \mathrm{GeV}$ electron beam and $0.7-6.0 \% L_{R}$ gold targets. Whereas in the first area the discrepancy between the LPM theory predictions and data is about 3.2 to $5 \%$ that requires the use of normalization factor $0.94 \pm 0.01 \pm 0.032$, in the second area this discrepancy reaches $\sim 15 \%$.

Using the second-order representation of the Molière distribution function (14), (15) for computing the spectral radiation rate (56) the authors of [40] were able to agree satisfactorily theory and $25 \mathrm{GeV}$ and $0.7 \% L_{R}$ data over the range $\omega<30 \mathrm{MeV}$.

This result can be understood by considering the fact that the correction to the Gaussian first-order representation of the distribution function $w_{M}(\vartheta)$ of order of $1 / B^{B}$ is about $12 \%$ for the used in calculations value $B^{B}=8.46[40]$.

\subsection{Coulomb corrections in the coherent radi- ation theory for a thin target}

Let us obtain the relative Coulomb correction to the average value of the spectral density of radiation for two limiting cases (59).

In the first case $\gamma^{2} \overline{\vartheta^{2}} \ll 1$, takin into account the equality

$$
\delta_{C C}\left[\gamma^{2} \overline{\vartheta^{2}}\right]=\delta_{C C}\left[\overline{\vartheta^{2}}\right]
$$

(47), and (59), we get

$$
\delta_{C C}\left[\left\langle\frac{d I}{d \omega}\right\rangle\right]=\delta_{C C}\left[\left(\frac{d I}{d \omega}\right)_{0}\right]=\frac{f(\xi)}{1-B^{B}},
$$

where $B^{B} \approx 8.46$ in the conditions of the discussed experiment [40].

In the second case $\gamma^{2} \overline{\vartheta^{2}} \gg 1$, we have

$$
\begin{gathered}
\Delta_{C C}\left[\ln \left(\gamma^{2} \overline{\vartheta^{2}}\right)-1\right]=\Delta_{C C}\left[\ln \left(\overline{\vartheta^{2}}\right)\right] \\
=\Delta_{C C}[\ln (B)] .
\end{gathered}
$$

For the latter quantity one can obtain

$$
\Delta_{C C}[\ln (B)]=\Delta_{C C}[B]+f(Z \alpha)=\delta_{C C}[B] .
$$

The Coulomb correction then becomes

$$
\Delta_{C C}\left[\ln \left(\gamma^{2} \overline{\vartheta^{2}}\right)-1\right]=\frac{\delta_{C C}[B]}{\left[\ln \left(\gamma^{2} \overline{\vartheta^{2}}\right)^{B}-1\right]} .
$$

Taking into account (47), we arrive at a result:

$$
\delta_{C C}\left[\left\langle\frac{d I}{d \omega}\right\rangle\right]=\frac{f(\xi)}{\left[\ln \left(\gamma^{2} \overline{\vartheta^{2}}\right)^{B}-1\right]\left(1-B^{B}\right)} .
$$

The numerical values of these corrections are presented below.

Table 6. The relative Coulomb correction $\delta_{C C}[\langle d I / d \omega\rangle]$ to the asymptotes of the Born spectral radiation rate over the range $\omega<\omega_{c}$ for $\beta=1$,

$$
B^{B} \approx 8.46 \text {, and }\left(\gamma^{2} \overline{\vartheta^{2}}\right)^{B} \approx 7.61[40] .
$$

\begin{tabular}{lcccc}
\hline Target & $\mathrm{Z}$ & $\gamma^{2} \overline{\vartheta^{2}}$ & $-\delta_{C C}[\langle d I / d \omega\rangle]$ & $R_{C C}$ \\
\hline $\mathrm{Au}$ & 79 & $\gamma^{2} \overline{\vartheta^{2}} \ll 1$ & 0.042 & 0.958 \\
$\mathrm{Au}$ & 79 & $\gamma^{2} \overline{\vartheta^{2}} \gg 1$ & 0.040 & 0.960 \\
\hline
\end{tabular}

The second asymptote is not reached [40] in the conditions of experiment $[27,28]$. Therefore we will also consider an another limiting case corresponding to these experimental conditions and taking into account the second term of the Molière distribution function expansion (9).

Inserting the second-order expression (14) for the distribution function into (56) and integrating its second 
term (15), we can arrive at the following expression for the electron radiation spectrum at $\mu^{2}=\gamma^{2} \overline{\vartheta^{2}} \gg 1[40]$ :

$$
\left\langle\frac{d I}{d \omega}\right\rangle=\frac{2 e^{2}}{\pi}\left[\ln \left(\mu^{2}\right)-C_{E}\left(1+\frac{2}{\mu^{2}}\right)+\frac{2}{\mu^{2}}+\frac{C_{E}}{B}-1\right]
$$

In order to obtain the Coulomb correction to the Born spectral radiation rate from $(66)$, we first calculate its numerical value at $\left(\mu^{2}\right)^{B} \approx 7.61$ and $B^{B} \approx 8.46$. Then we become $\langle d I / d \omega\rangle^{B}=0.00542$. The Bethe-Heitler formula in the Born approximation gets $\langle d I / d \omega\rangle_{B H}^{B}=$ 0.00954 .

Now we calculate the numerical values of $B$ and $\mu^{2}$ parameters including the Coulomb corrections. From

$$
\Delta_{C C}[B]=\frac{f(\xi)}{1 / B^{B}-1}=-0.355
$$

we become $B=8.105$ for $Z=79$ and $B^{B} \approx 8.46$. The equality

$$
\begin{aligned}
\Delta_{C C}\left[\ln \mu^{2}\right] & =\Delta_{C C}[\ln B]=\Delta_{C C}[B]+f(\xi) \\
& =\delta_{C C}[B]=-0.042
\end{aligned}
$$

gets $\ln \mu^{2}=1.987$ and $\mu^{2}=7.295$. Inserting these values into $(66)$ we have $\langle d I / d \omega\rangle=0.00531$. The relative Coulomb correction to these parameters presents Table 7 . These corrections are not large. Their sizes are between two to four percent, i.e. of order of the systematic error in the experiment [27].

Table 7. The relative Coulomb corrections in the analogue of the LPM effect theory for $0.07 L_{R}$ gold target, $\omega<\omega_{c}$, and $\beta=1$.

\begin{tabular}{cccc}
\hline$\delta_{C C}\left[\ln \mu^{2}\right]$ & $\delta_{C C}\left[\left(\frac{d I}{d \omega}\right)_{0}\right]$ & $\delta_{C C}\left[\left\langle\frac{d I}{d \omega}\right\rangle\right]$ & $\delta_{C C}[\Phi(s)]$ \\
\hline-0.021 & -0.042 & -0.020 & -0.021 \\
\hline
\end{tabular}

Accounting the relative Coulomb correction to the Bethe-Heitler spectrum of bremsstrahlung we find $(d I / d \omega)_{B H}=0.00916$. So we get $^{6}$

$$
\left\langle\frac{d I}{d \omega}\right\rangle=0.580\left(\frac{d I}{d \omega}\right)_{B H} .
$$

This leads to the value of the spectral radiation rate in terms of $d N /[d(\log \omega)] \times 1 / L_{R}$, where $N$ is the number of events per photon energy bin per incident electron, $d N /\left[d(\log \omega) / L_{R}\right]=0.118 \times 0.580=0.068$, which agrees very well with the experimental result over the frequency range $\omega<30 \mathrm{MeV}$ for $25 \mathrm{GeV}$ and $0.7 \% L_{\mathrm{R}}$ gold target. This result additionally improves the agreement between the theory [39, 40] and experiment [27, 28] (see Fig. 2) and coincides with the result of [33] obtained in the eikonal approximation (Fig. 20a in [29]).

\footnotetext{
${ }^{6}$ The obtained magnitude $\Phi(s)=0.580$ corresponds to the value $s \sim 0.15$ (see Fig. 1 in [16]).
}

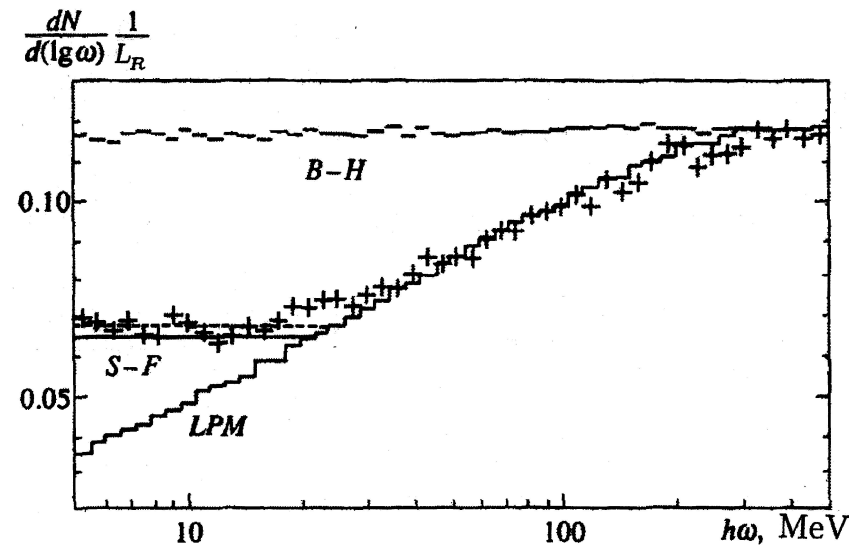

Figure 2. Measurement of the LPM effect over the range $30<\omega<300 \mathrm{MeV}$ and its analogue in the range $5<\omega<30$ $\mathrm{MeV}$ for the $0.7 \% L_{R}$ gold target and $25 \mathrm{GeV}$ electron beam. The signs ' + ' denote the experimental data; the histograms $B-H$ and $L P M$ give the Bethe-Heitler and the LPM Monte Carlo predictions from [27]. The solid and dashed lines over the range $\omega<30 \mathrm{MeV}$ are the results of calculations without [40] and with the Coulomb corrections obtained in this paper. Adapted from [40].

\section{Summary and conclusions}

1. We have calculated the Coulomb corrections $\Delta_{C C}[b], \quad \Delta_{C C}[B], \quad \Delta_{C C}[\ln B], \quad \Delta_{C C}\left[\overline{\vartheta^{2}}\right]$, $\Delta_{C C}\left[\ln \left(\overline{\vartheta^{2}}\right)\right]$ and relative Coulomb corrections $\delta_{C C}\left[\overline{\vartheta^{2}}\right]=\delta_{C C}[B]$ to some important parameters of the Molière multiple scattering theory for high $\mathrm{Z}$ targets of experiment $[27,28]$ and showed that the corrections $-\Delta_{C C}[b],-\Delta_{C C}[B]$ have a large value which increases up to $0.40-0.45$ for $Z=92$.

2. Using these corrections we have obtained the analytical results for the Coulomb corrections to the quantities of the Migdal LPM theory $\Delta_{C C}\left[(d I / d \omega)_{0}\right], \Delta_{C C}[q], \Delta_{C C}\left[s^{2}\right], \Delta_{C C}[s]$, $\Delta_{C C}\left[s^{4}\right], \Delta_{C C}[\Phi(s)]$, and $\Delta_{C C}[\langle d I / d \omega\rangle]$ in regimes of the large and the small LPM suppression.

3. We calculated relative Coulomb corrections $\delta_{C C}\left[(d I / d \omega)_{0}\right]=\delta_{C C}[q]$ for $s \gg 1$ and also $\delta_{C C}[\Phi(s)]=\delta_{C C}[s]$ together with $\delta_{C C}[\langle d I / d \omega\rangle]$ in the regime of large LPM suppression over the range $74 \leq Z \leq 92$ and demonstrated that the latter correction amounts to $-8 \%$ for $Z=92$.

4. We have also performed the calculations for the regime of small LPM suppression in the entire range $1 \leq s \leq \infty$. We found that the Coulomb corrections $\delta_{C C}[\langle d I / d \omega\rangle]=-4.50 \pm 0.05 \%(Z=82)$ and $\delta_{C C}[\langle d I / d \omega\rangle]=-5.35 \pm 0.06 \%(Z=92)$ coincides with the sizes of the normalization corrections $-4.5 \pm 0.2 \%$ for $2 \% L_{R}$ lead target and $-5.6 \pm 0.3 \%$ for $3 \% L_{R}$ uranium target, respectively, within the experimental error.

5. The average $\delta_{C C}[\langle d I / d \omega\rangle]$ value $\bar{\delta}_{C C}[\langle d I / d \omega\rangle]=$ $-4.70 \pm 0.49 \%$ excellent agrees in the regime of small LPM suppression with the weighted average 
$-4.7 \pm 2 \%$ of the normalization correction obtained for $25 \mathrm{GeV}$ data in the experiment [28].

6. Thus, we managed to show that the discussed discrepancy between theory and experiment can be explained both qualitatively and quantitatively on the basis of the obtained Coulomb corrections to the Born bremsstrahlung rate for heavy target elements within the Migdal LPM effect theory.

7. We found that applying the revised Molière multiple scattering theory allows to avoid multiplying theoretical results by above normalization factor and leads to agreement between the theory of LPM effect and experimental data $[27,28]$ for high $\mathrm{Z}$ targets over the range $20<\omega<500 \mathrm{MeV}$.

8. Finally, we found the numerical values of the relative Coulomb corrections $\delta_{C C}\left[(d I / d \omega)_{0}\right], \delta_{C C}[\Phi(s)]$, and $\delta_{C C}[\langle d I / d \omega\rangle]$ in the LPM effect theory analogue for thin targets over the range $5<\omega<30$ and demonstrated that these corrections additionally improves the agreement between the theory $[39,40]$ and experiment $[27,28]$.

9. The approach based on application of the improved Molière multiple scattering theory can be useful to the analysis of electromagnetic processes in strong crystalline fields at high energies $(\sim 300 \mathrm{GeV}$ electron beams) [31], in describing cosmic ray experiments, the high energy experiments with nuclear targets (MUSCAT, MUCOOL experiments, the DIRAC experiment at CERN), etc.

\section{Acknowledgements}

We would like to thank Spencer Klein (Lawrence Berkeley National Laboratory, USA) for valuable information and illuminating discussion.

\section{REFERENCES}

[1] S. A. Goudsmit, J. L. Saunderson. Phys. Rev., 57, 24 (1940); 58, 36 (1940).

[2] B. Rossi, K. Greisen. Rev. Mod. Phys. 13, 240 (1941).

[3] G. Molière. Z. Naturforsch., 2 a, 133 (1947); 3 a, 78 (1948); 10 a, 177 (1955).

[4] H. Snyder, W. T. Scott. Phys. Rev., 76, 220 (1949); W. T. Scott, Phys. Rev., 85, 245 (1952).

[5] H. A. Bethe. Phys. Rev., 89, 256 (1953).

[6] U. Fano. Phys. Rev., 93, 117 (1954).

[7] R. J. Glauber. Phys. Rev., 100, 242 (1955); R. J. Glauber. 1959 Lectures in Theoretical Physics, 1, Eds. W. Brittain, L. Dunham, New York, 1959, p. 315.

[8] DIDAC-Collaboration: B. Adeva, L. Afanasyev, M. Benayoun et al. Phys. Lett., B 704, 24 (2011); B 619, 50 (2005); A. Dudarev et al. DIRAC note 2005-02.

[9] N. O. Elyutin et al. Instrum. Exp. Tech., 50, 429 (2007); J. Surf. Invest., 4, 908 (2010).
[10] MuScat Collaboration: D. Attwood et al. Nucl. Instrum. Meth., B 251, 41 (2006); A. Tollestrup. J. Monroe. NFMCC technical note MC-176, September 2000.

[11] R. C. Fernow. MUC-NOTE-COOL theory-336, April 2006; A. Van Ginneken. Nucl. Instr. Meth., B 160, 460 (2000); C. M. Ankebrandt et al. Proposal of the MUCOOL Collaboration, April 2012.

[12] S. I. Striganov. Radiat. Prot. Dosimetry, 116, 239 (2005); A. V. Butkevich, R. P. Kokoulin, G. V. Matushko et al. Nucl. Instrum. Meth. Phys. Res., A 488, 282 (2002); V. I. Yurchenko. JETP, 89, 223 (1999).

[13] F. S. Dzheparov et al. JETP Lett., 72, 518 (2000); 78, 1011 (2003); J. Surf. Invest., 3, 665 (2009).

[14] L. D. Landau, I. Ya. Pomeranchuk. Dokl. Akad. of Science of SSSR, 92, 535 (1953); 92, 735 (1953) (in Russian) [Translated into English: In The Collected Papers of L. D. Landau, New-York, Pergamon Press, 1965, p. 589].

[15] A. B. Migdal. Dokl. Akad. of Science of SSSR, 96, 49 (1954).

[16] A. B. Migdal. Phys. Rev., 103, 1811 (1956); JETP, 32, 633 (1957).

[17] I.I. Goldman. JETP, 38, 1866 (1960).

[18] N. F. Shul'ga, S. P. Fomin. JETP. Lett., 27, 117 (1978); Probl. Atom. Sci. Technol., 2, 11 (2003); N. F. Shul'ga. Int. J. Mod. Phys., A 25, 9 (2010).

[19] L. Gerhardt, S. R. Klein. Phys. Rev., D 82, 074017 (2010).

[20] IceCube Collaboration: R. Abbasi R. et al. Phys. Rev. Lett., 111, 021103 (2013); S. R. Klein. Nucl. Phys. (Proc. Suppl.), B 00, 1 (2010).

[21] M. J. van Goethem, L. Aphecetche, J. C. S. Bacelar et al. Phys. Rev. Lett., 88, 122302 (2002).

[22] A. H. Sørensen. Z. Phys., C 53, 1609 (1992); S. J. Brodsky, P. Hoyer. Phys. Lett., B 298, 165 (1993); B. Z. Kopeliovich, A. Schäfer, A. V. Tarasov. Phys. Rev., C 59, 1609 (1999).

[23] M. Gyulassy, X.-N. Wang. Nucl. Phys., B 420, 583 (1994); R. Baier, Yu. L. Dokshitzer, S. Peignè et al. Phys. Lett., B 345, 277 (1995); B.G. Zakharov. JETP Lett., 63, 952 (1996).

[24] V. N. Baier, V. M. Katkov. Phys. Lett., A 353, 91 (2006).

[25] G. Raffelt, D. Seckel. Phys. Rev. Lett., 67, 2605 (1991); C.J. Pethick, V. Thorsson. Phys. Rev. Lett., 72, 1964 (1994).

[26] S. Peigne, A. V. Smilga. Advan. Phys. Sci., 179, 697 (2009).

[27] P. L. Anthony, R. Becker-Szendy, P. E. Bosted et al. Phys. Rev. Lett., 75, 1949 (1995); 76, 3550 (1996).

[28] P. L. Anthony, R. Becker-Szendy, P. E. Bosted et al. Phys. Rev., D 56, 1373 (1997).

[29] S. R. Klein. Rev. Mod. Phys., 71, 1501 (1999). 
[30] H. D. Hansen, U. I. Uggerhoj, C. Biino et al. Phys. Rev. Lett., 91, 014801 (2003); Phys. Rev., D 69, 032001 (2004).

[31] J. U. Andersen, K. Kirsebom, S. P. Moller et al. Electromagnetic Processes in Strong Cristalline Fields, CERNSPSC-2005-030; CERN-NA63 Collaboration. Phys. Lett., B 672, 323 (2009); Nucl. Instrum. Meth., B 268, $1412(2010)$

[32] B. G. Zakharov. JETP Lett., 64, 781 (1996).

[33] R. Blancenbeckler, S. D. Drell. Phys. Rev., D 53, 6265 (1996); R. Blancenbeckler. Phys. Rev., D 55, 190 (1997)

[34] V. N. Baier, V. M. Katkov. Phys. Rev., D 57, 3146 (1998).

[35] B. G. Zakharov. Yad. Fiz., 62, 1075 (1999).

[36] N. V. Laskin, A. S. Mazmanishvili, N. F. Shul'ga. Dokl. Akad. of Science of SSSR, 277, 850 1984; Phys. Lett., A 112, 240 (1985); A. I. Akhiezer, N. V. Laskin, N. F. Shul'ga. Dokl. Akad. of Science of SSSR., 295, 1363 (1987).
[37] A. V. Tarasov, O. O. Voskresenskaya. In: Alexander Vasilievich Tarasov: To the 70th Birth Anniversary, Dubna: JINR, 2012. p. 276 [ArXiv:1107.5018 v 2, hep-ph]; A. V. Tarasov, O. O. Voskresenskaya. ArXiv:1204.3675, hep-ph.

[38] E. A. Kuraev, O. O. Voskresenskaya, A. V. Tarasov. JINR E2-2012-135, Dubna, 2012 (submitted to Phys. Rev. Lett.).

[39] N. F. Shul'ga, S. P. Fomin. JETP. Lett., 63, 873 (1996).

[40] N. F. Shul'ga, S. P. Fomin. JETP. Lett., 113, 58 (1998).

[41] W. T. Scott. Rev. Mod. Phys., 35, 231 (1963).

[42] R. P. Feynman, A. R. Hibbs. Quantum mechanics and path integrals, New York, McGrav-Hill, 1965.

[43] M. L. Ter-Mikaelian. Zh. Eksp. Teor. Fiz., 25, 289 (1953); 25, 296 (1953); High Energy Electromagnetic Processes in Condensed Media, New York, Wiley Interscience, 1972. 